\title{
COMPARISON RESULTS AND LINEARIZED OSCILLATIONS FOR HIGHER-ORDER DIFFERENCE EQUATIONS
}

\author{
G. LADAS \\ Department of Mathematics \\ The University of Rhode Island \\ Kingston, R.I.02881-0816, U.S.A. \\ and \\ C. QIAN \\ Department of Mathematics \\ The University of Rhode Island \\ Kingston, R.I.02881-0816, U.S.A. \\ and \\ Department of Mathematics \\ Yangzhou Teacher's College \\ Yangzhou, Jiangsu, P.R.C.
}

(Recelved May 8, 1989 and in revised form February 20, 1990)

ABSTRACT. Consider the difference equations

$$
\Delta^{m} x_{n}+(-1)^{m+1} p_{n} f\left(x_{n-k}\right)=0, n=0,1, \ldots
$$

and

$$
\Delta^{m} y_{n}+(-1)^{m+1} q_{n} \varepsilon\left(y_{n-l}\right)=0, n=0,1, \ldots .
$$

We establish a comparison result according to which, when $m$ is odd, every solution of Eq.(1) oscillates provided that every solution of Eq.(2) oscillates and, when m is even, every bounded solution of Eq.(1) oscillates provided that every bounded solution of Eq.(2) oscillates. We also establish a linearized oscillation theorem accordino to which, when $m$ is odd, every solution of Eq.(1) oscillates if and only if every solution of an associated linear equation

$$
\Delta^{m} z_{n}+(-1)^{n+1} p z_{n-k}=0, n=0,1, \ldots
$$

oscillates and, when $m$ is even, every bounded solution of Eq.(1) oscillates if and only if every bounded solution of $(*)$ oscillates.

KEY WORDS AND PHRASES. Linearized oscillations, Higher order difference equations, Comparison results.

1980 AMS SUBJECT CLASSIFICATION CODE. 39 A12

\section{INTRODUCTION}

Let $\boldsymbol{\square}=\{0,1,2, \ldots\}$ denote the set of nonnegative integers, let $\Delta$ be the first order forward difference operator, $\Delta x_{n}=x_{n+1}-x_{n}$ and for $m \stackrel{1}{=}$, let $\Delta^{m}$ be the $m^{\text {th }}$ 
order forward difference operator, $\Delta^{m} x_{n}=\Delta\left(\Delta^{m-1} x_{n}\right)$.

Consider the difference equations

$$
\Delta^{m} x_{n}+(-1)^{m+1} p_{n} f\left(x_{n-k}\right)=0, n=0,1, \ldots
$$

and

$$
\Delta^{m} y_{n}+(-1)^{m+1} q_{n} g\left(y_{n-\ell}\right)=0, n=0,1, \ldots
$$

where

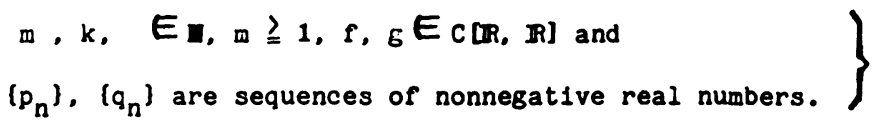

Our aim in this paper is to establish a comparison result according to which, when $m$ is odd, if every solution of Eq.(1.2) oscillates, then so does every solution of Eq.(1.1) and, when $m$ is even, if every bounded solution of Eq.(1.2) oscillates then so does every bounded solution of Eq.(1.1). We will also establish a linearized oscillation theorem according to which, when $m$ is odd, every solution of Eq.(1.1) oscillates if and only if every solution of an associated linear equation with constant coefricient $p \in(0, \infty)$,

$$
\Delta^{m} z_{n}+(-1)^{m+1} p z_{n-k}=0, n=0,1, \ldots
$$

oscillates, and when $m$ is even, every bounded solution of Eq.(1.1) oscillates if and only if every bounded solution of Eq.(1.4) oscillates.

By a solution of Eq.(1.1) we mean a sequence $\left(x_{n}\right)$ which is defined for $n \geq-k$ and which satisfies $E q .(1.1)$ for $n=0,1, \ldots$. Clearly, if

$$
a_{-k}, \ldots a_{0}, \ldots, a_{m-1}
$$

are given real numbers, then Eq.(1.1) has a unique solution $\left[x_{n}\right)$ satisfying the initial conditions

$$
x_{n}=a_{n} \text { for } n=-k, \ldots, 0, \ldots m-1 \text {. }
$$

When $k E$. Eq. (1.1) is a difference equation of order $(k+m)$. However, one may also think of Eq.(1.1) as being an $\mathrm{m}^{\text {th }}$ order delay difference equation with delay $(k+k 1)$. When $k$ is a nezative integer. Eq.(1.1) is a difference equation of order equal to $\max \{-k, m\}$ but one may also think of Eq.(1.1) as being an $m^{\text {th }}$ order advanced difference equation.

A solution $\left\{x_{n}\right\}$ of Eq.(1.1) is said to oscillate if for every $n_{0} ? 0$ there exists an $n \geqq n$. such that

$$
x_{n} x_{n+1} \leqq 0
$$

Otherwise the solution is called nonoscillatory.

Recently there has been a lot of interest in the oscillations of delay difference equations. See, for example, $[1]-[3],[5]-[7]$ and [10]. For the oscillation of second order difference equations see [4], [8] and [9] and the references cited therein.

\section{SOME BASIC LEMMAS}

In this section we will present some basic lemmas which are interesting in their own rights and which will be used in the proofs of the main theorems in Sections 3, 4 and 5. The first lemma is a special case of a general result which was established in 
[3], see also [10].

LEMMA 1. Assune that

$q \in \mathbb{R}$ and $k \in \mathbb{Z}=\{\ldots,-2,-1,0,1,2, \ldots\}$.

Then every solution of the difference equation

$$
\Delta u_{n}+q u_{n-k}=0
$$

oscillates if and only if the characteristic equation

$$
(\lambda-1)^{m}+q \lambda^{-k}=0
$$

has no positive roots.

By investigating the conditions under which Eq:(2.3) has no positive roots one is led to the following corollary of Lemma 1 which gives explicit necessary and sufficient conditions for the oscillation of all solutions of Eq.(2.2).

COROLLARY 1. Assume that (2.1) holds. Then every solution of Eq.(2.2) oscillates if and only if one of the following conditions is satisfied:

(i) $m$ is even and $q>0$;

(ii) $m$ is odd, $k=0$ and $q \stackrel{1}{=}$;

(iii) $\mathrm{a}$ is odd, $k=-m$ and $q \leqq-1$;

(iv) $m$ is odd, $k>0$ or $k\left\langle-m\right.$, and $\left.q \frac{(k+m)^{k+m}}{k^{k}}\right\rangle m^{m}$.

Concerning the oscillation of all bounded solutions of Eq.(2.2) one can see that the following result holds.

LEMMA 2. Assure that (2.1) holds. Then every bounded solution of Eq.(2.2) oscillates if and only if Eq. (2.3) has no roots in $(0,1)$.

By investigating the conditions under which Eq.(2.3) has no roots in $(0,1]$ one is led to the following corollary of Lemma 2 .

COROLLARY 2. Assume that (2.1) holds. Then every bounded solution of Eq. (2.2) oscillates if and only if one of the following condition is satisfied:

(i) $m$ is even and $q>0$;

(ii) in is even, $q E(-\infty,-1)$ and $k=0$;

(iii) w is even, $q \in\left(-\infty,-\frac{m^{m} k^{k}}{(k+m)^{k+m}}\right)$ and $k \stackrel{\geq}{=}$;

(iv) $m$ is odd and $q<0$;

(v) $m$ is odd, $q \in[1, \infty)$ and $k=0$;

(vi) $m$ is odd, $q \in\left(\frac{m^{m} k}{(k+m)^{k+m}}, \infty\right)$ and $k \geq 1$.

LEMriA 3. Assume that every solution of Eq.(2.2) oscillates and that one of the following three conditions is satisfied:

(i) $q \in(0, \infty)$ and $k \in\{1,2, \ldots\}$;

(ii) $q \in(1, \infty), k=0$ and $m$ is odd;

(iii) $q \in(0, \infty), k=0$ and $m$ is even.

Then there exists an $\varepsilon_{0} E(0, q / 2)$ such that for every $E\left[0, \varepsilon_{0}\right]$ every solution of the delay difference equation 


$$
\Delta^{m} u_{n}+(q-\varepsilon) u_{n-k}=0
$$

also oscillates.

PROOF. In view of Lema 1 it suffices to show that the characteristic equation of Eq.(2.7) has no positive roots. The hypothesis that every solution of Eq.(2.2) oscillates implies that the characteristic equation

$$
F(\lambda)=(\lambda-1)^{m}+q \lambda^{-k}=0
$$

of Eq. (2.2 has no positive roots. As $F(\infty)=\infty$ it follows that

$$
F(\lambda)>0 \text { for all } \lambda E_{R^{+}} \text {. }
$$

First assume that $(2.4)$ holds. From $(2.8)$ we obtain, $F\left(0^{+}\right)=F(\infty)=\infty$. Hence

$$
\mu=\min [F(\lambda): 0<\lambda<\infty]
$$

exists and is a positive number and so

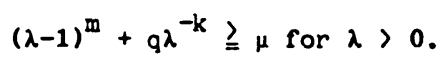

Set $\delta=q / 2$ and choose $0<\lambda_{1}<\lambda_{2}$ such that

$$
(\lambda-1)^{m}+\frac{9}{2} \lambda^{-k}>0 \text { for } \lambda \in\left(0, \lambda_{2}\right) \cup\left(\lambda_{2}, \infty\right) \text {. }
$$

Let

$$
\eta=\max \left(\lambda^{-k}: \lambda_{1} \leqq \lambda \leqq \lambda_{2}\right)
$$

and set

$$
\varepsilon_{0}=\min \left(\delta, \frac{\mu}{2 \eta}\right) \text {. }
$$

Now let $0 \leqq e \leqq \varepsilon_{0}$. Then for $\lambda E\left(0, \lambda_{2}\right) U\left(\lambda_{2}, \infty\right)$,

$$
(\lambda-1)^{m}+(q-\varepsilon) \lambda^{-k} \geqq(\lambda-1)^{m}+\frac{q}{2} \lambda^{-k}>0 \text {. }
$$

On the other hand for $\lambda_{1} \leqq \lambda \leqq \lambda_{2}$.

$$
(\lambda-1)^{m}+(q-\varepsilon) \lambda^{-k} \geqq m-\varepsilon_{0} \eta \geqq m-\frac{m}{2}>0 .
$$

The proof when $(2.4)$ holds is complete.

Next assurie that $(2.5)$ holds. Set $e_{0}=\frac{q-1}{2}$. Then for $\lambda \geqq 1$ and $0 \leqq \varepsilon \leqq \varepsilon_{0}$.

$$
(\lambda-1)^{m}+q-\varepsilon \stackrel{?}{\underline{m}} q-\varepsilon_{0}=\frac{q+1}{2}>0
$$

while for $\lambda<1$ and $0 \leqq \varepsilon \leqq \varepsilon_{\bullet}$.

$$
(\lambda-1)^{m}+q-\varepsilon \geqq-1+q-\varepsilon_{0}=\frac{q-1}{2}>0 .
$$

Finally when (2.6) holds, one can see that $e_{0}=q / 2$ is a good choice. The proof is complete.

By using Lemma 2 and by an argument similar to that in the proof of Lemma 3 we can easily establish the following result. The proof will be omitted.

LEMMA 4. Assume that every bounded solution of Eq.(2.2) oscillates and that one of the following three conditions is satisfied:

(i) $q \in(-\infty, 0)$ and $k \in\{1,2, \ldots\}$;

(ii) $q \in(-\infty, 0), k=0$ and $m$ is odd;

(iii) $q E(-\infty,-1), k=0$ and $m$ is even.

Then there exists an $\varepsilon_{0} E(0,-q / 2)$ such that for every $\varepsilon E\left[0, \varepsilon_{0}\right]$ every bounded solution of the delay difference equation 


$$
\Delta^{a} u_{n}+(q+\varepsilon) u_{n-k}=0
$$

also oscillates.

LEMMA 5. Assume that $h \in C[R, \mathbb{R}], u h(u)>0$ for $u \neq 0$, and $h(u)$ is nondecreasins in a neighborhood of the origin. Let $\left\{r_{n}\right\}$ be a sequence of nonneüative real numbers and

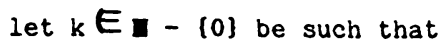

$$
\sum_{i=n+1}^{n+k} r_{i}>0 \text { for } n=0,1, \ldots .
$$

Suppose that the difference inequality

$$
\sum_{i_{1}=n}^{\infty} \sum_{i_{2}=i_{1}}^{\infty} \ldots \sum_{i_{m}=i_{m-1}}^{\infty} r_{i_{m}} h\left(y_{i_{m}-k}\right) \leqq y_{n,} n \geqq N
$$

has a positive solution $\left\{y_{n}\right\}$ defined for $n \geqq N-k$ and such that

$$
\lim _{n \rightarrow \infty} y_{n}=0 \text {. }
$$

Then there exists a positive solution $\left(x_{n}\right)$, defined for $n \geqq N-k$, of the correspondins difference equation

$$
\sum_{i_{1}=n}^{\infty} \sum_{i_{2}=i_{1}}^{\infty} \ldots \sum_{i_{m}=i_{m-1}}^{\infty} r_{i_{m}} h\left(x_{i_{m}-k}\right)=x_{n}, n ! N
$$

which satisfies

$$
\lim _{n \rightarrow \infty} x_{n}=0 \text {. }
$$

PROOF. Choose a positive number $\delta$ and an integer $N^{\prime} \geqq N$ such that

$$
\begin{aligned}
& h(u) \text { is nondecreasing in }[0, \delta], \\
& y_{n}>y_{N} \text { for } N-k \leqq n<N^{\prime} \text { and } 0<y_{n}<\delta \text { for } n \geqq N^{\circ}-m .
\end{aligned}
$$

Define the set of nonnegative sequences

$$
x=\left\{x=\left\{x_{n}\right\}: 0 \leqq x_{n} \leqq y_{n} \text { for } n \geqq N^{\prime}\right\}
$$

and for every $x \in X$ define the sequence $x=\left\{x_{n}\right\}$ by

$$
x_{n}=\left\{\begin{array}{l}
x_{n} \text { for } n \geqq N^{\prime} \\
x_{N^{\prime}}+y_{n}-y_{N^{\prime}}, \text { for } N-k \leqq n<N^{\prime} .
\end{array}\right.
$$

Clearly

$$
0 \leqq x_{n} \leqq y_{n} \text { for } n \geqq N-k
$$

and

$$
\bar{x}_{\mathrm{n}}>0 \text { for } \mathrm{N}-\mathrm{k} \leqq \mathrm{n}<\mathrm{N} \text {. }
$$

Define the mapping $T$ on $X$ as follows: For every $x=\left\{x_{n}\right\} E X$ let the $n^{\text {th }}$ term of the sequence $T x$ be

$$
\sum_{i_{1}=n}^{\infty} \sum_{i_{2}=i_{1}}^{\infty} \ldots \sum_{i_{m}=i_{m-1}}^{\infty} r_{i_{m}} h\left(\bar{x}_{i_{m}-k}\right) .
$$

It is clear that $T$ is monotone in the sense that if $x^{(1)}, x^{(2)} \in x_{\text {and }} x^{(1)} \leqq x^{(2)}$ (that is, $x_{n}{ }^{(1)} \leqq x_{n}{ }^{(2)}$ for $n \geqq N^{\prime}$ ) then $T x{ }^{(1)} \leqq T x{ }^{(2)}$. Note that $T y \leqq y$ and so $x \in X$ implies $T x \leqq T y \leqq y$. Thus $T: X \rightarrow X$. We now define the following sequence on $X$ :

$$
x^{(0)}=\left\{y_{n}\right\} \text { for } n \geqq N^{\prime} \text { and } x^{(s)}=T x^{(s-1)} \text { for } s=1,2, \ldots \text {. }
$$


It follows by induction that the sequence $\left(x^{(s)}\right)$ of elements of $X$ is such that

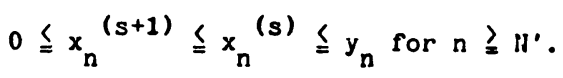

Hence

$$
x_{n}=\lim _{s \rightarrow \infty} x_{n}^{(s)}, n \geqq N^{\prime}
$$

exists and $x=\left\{x_{n}\right\}_{n=N}^{\infty}$, belongs to $X$. Also $x=T x$ and so $x$ is a solution of Eq. (2.12). Furthermore (2.13) holds. It remains to show that

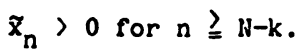

Otherwise, in view of (2.15), there exists an $s ? N^{\prime}$ such that

$$
\tilde{x}_{s}=0 \text { and } \tilde{x}_{n}>0 \text { for } N-k \leqq n \leqq s-1 .
$$

Then from $(2.12)$,

$$
\sum_{i_{1}=s}^{\infty} \sum_{i_{2}=i_{2}}^{\infty} \cdots \sum_{i_{m}=i_{m-1}}^{\infty} r_{i_{m}} h\left(\tilde{x}_{i_{m}-k}\right)=\tilde{x}_{s}=0 .
$$

But by $(2.9)$ and $(2.16)$.

$$
\sum_{i_{1}=s}^{\infty} \sum_{i_{2}=i_{1}}^{\infty} \ldots \sum_{i_{m}=i_{m-2}}^{\infty} r_{i_{m}} h\left(\tilde{x}_{i_{m}-k}\right) \geq \sum_{i=s}^{s+k-1} r_{i} h\left(\tilde{x}_{i-k}\right)>0 .
$$

This is a contradiction and the proof is complete.

LEMIA 6. Consider the difference equation

$$
\Delta^{m} x_{n}+p_{n} f\left(x_{n-k}\right)=0 \text {. }
$$

where

$$
\begin{aligned}
& k E n,\left\{p_{n}\right\} \text { is a nonnegative sequence of real numbers, } \\
& f \in c[[R, \mathbb{R}], u f(u)>0 \text { for } u \neq 0
\end{aligned}
$$

and there exist $\alpha, \beta E(0, \infty)$ such that

$$
|f(u)| \geq \text { a for }|u| \geq \beta \text {. }
$$

Suppose also that

$$
\sum_{i=0}^{\infty} p_{i}=\infty
$$

and let $\left\{x_{n}\right\}$ be a nonoscillatory solution of Eq.(2.17). Then eventually.

$$
x_{n}\left(\Delta^{m} x_{n}\right) \leqq 0,(-1)^{i+1} x_{n}\left(\Delta^{m-1} x_{n}\right)>0 \text { for } 1=1,2, \ldots . m
$$

and

$$
\lim _{n \rightarrow \infty}\left(\Delta^{i} x_{n}\right)=0 \text { for } i=0,1, \ldots m-1 \text {. }
$$

PROOF. We assume that $\left(x_{n}\right\}$ is an eventually positive solution of Eq.(2.17). The case where $\left(x_{n}\right)$ is eventually negative is similar and will be omitted. From Eq.(2.17),

$$
\Delta \Delta^{m} x_{n}=-p_{n} r\left(x_{n-k}\right) \leqq 0
$$

and so either

$$
\lim _{n \rightarrow \infty} \Delta^{m-1} x_{n}=-\infty
$$

or 


$$
\lim _{n \rightarrow \infty} \Delta^{m-1} x_{n}=\gamma E \mathbb{R}
$$

We claim that $(2.25)$ holds. Otherwise $(2.24)$ holds which implies that

$$
\lim _{n \rightarrow \infty} \Delta^{i} x_{n}=-\infty \text { for } i=0,1, \ldots,-1 \text {. }
$$

This contradicts the hypothesis that $\left\{x_{n}\right\}$ is positive and so (2.25) holds. From (2.23) and (2.25) it is easy to see that for each $i=0,1, \ldots, n-1$, the sequence $\left\{\Delta^{i} x_{n}\right\}$ is eventualiy monotonic. Now by suming both sides of (2.23) from $n_{1}$ to $\infty$, for $n_{1}$ sufficiently large, we obtain

$$
\gamma-\Delta^{m-1} x_{n_{1}}=-\sum_{i=n_{1}}^{\infty} p_{i} f\left(x_{i-k}\right) .
$$

As $\left(x_{n}\right)$ is eventualiy monotonic,

$$
\lim _{n \rightarrow \infty} x_{n}=\mu E[0, \infty]
$$

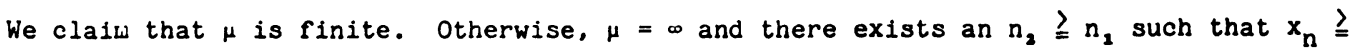
f for $n \stackrel{n}{=} n_{2}$. Then $\left(2.19^{\prime}\right)$ implies that

$$
f\left(x_{n}\right) \geq a>0 \text { for } n \geqq n_{2} \text {. }
$$

In view of $(2.20)$ and $(2.27),(2.26)$ is impossible and so $\mu E \mathbb{R}^{+}$. Then by the continunity of $f(u)$,

$$
\lim _{n \rightarrow \infty} f\left(x_{n}\right)=f(\mu)
$$

which, in view of (2.19), (2.20) and (2.26), implies that $\mu=0$. From this fact and the monotonicity of $\left\{\Delta^{i} x_{n}\right\}$ for $i=0,1, \ldots, m-1$ it follows that $(2.21)$ and (2.22) hold. The proof is complete.

RENARK 1. From the proof of Lemma 6 one can see that if $\left\{x_{n}\right\}$ is bounded then the conclusion of Lemma 6 is true without the condition (2.19').

COROLLARY 3. Assume that $m$ is even and that (2.18), (2.19), (2.19') and (2.20) hold. Then every solution of Eq.(2.17) oscillates.

PROOF. Assume, for the sake of contradiction, that Eq.(2.17) has a nonoscillatory solution $\left\{x_{n}\right\}$. Then for $n$ sufficiently large and for $1=m,(2.21)$ yields

$$
-\left(x_{n}\right)^{2}>0
$$

which is impossible and the proof is complete.

LEIMA 7. Assume that (2.18), (2.19) and (2.20) hold and assume that $\left(x_{n}\right)$ is a nonoscillatory solution of the difference equation

$$
\Delta^{m} x_{n}-p_{n} f\left(x_{n-k}\right)=0, n=0,1, \ldots .
$$

Then the following statements are true:

(i) if $\left\{x_{n}\right\}$ is bounded then

$$
x_{n}\left(\Delta^{m} x_{n}\right) \geqq 0,(-1)^{i} x_{n}\left(\Delta^{m-i} x_{n}\right)>0 \text { for } 1=1,2, \ldots . m
$$

and

$$
\lim _{n \rightarrow \infty}\left(\Delta^{i} x_{n}\right)=0 \text { for } i=0,1, \ldots, \text { nn-1. }
$$

(ii) if $\left(x_{n}\right)$ is unbounded and $\left(2.19^{\prime}\right)$ holds then 


$$
\lim _{n \rightarrow \infty}\left(\Delta^{i} x_{n}\right)=\omega \text { for } i=0,1, \ldots, n-1 .
$$

PROOF. He assume that $\left(x_{n}\right\}$ is an eventually positive solution of Eq.(2.28). The case where $\left(x_{n}\right)$ is eventually negative is similar and will be omitted. From Eq.(2.28) we see that eventually,

$$
\Delta^{m} x_{n}=p_{n} f\left(x_{n-k}\right) \geqslant 0 .
$$

and so either

$$
\lim _{n \rightarrow \infty} \Delta^{m-1} x_{n}=\infty
$$

or

$$
\lim _{n \rightarrow \infty} \Delta^{m-1} x_{n}=\gamma \in \mathbb{R} \text {. }
$$

Hence for each $1=0,1, \ldots, m-1,\left(\Delta^{i} x_{n}\right)$ is monotonic and so

$$
\lim _{n \rightarrow \infty} x_{n}=\mu E[0, \infty] \text {. }
$$

By summing both sides of (2.32) from $n_{2}$ to $n$ we obtain

$$
\Delta^{m-1} x_{n}-\Delta^{m-1} x_{n_{1}}=\sum_{i=n_{1}}^{n} p_{i} f\left(x_{i-k}\right) \text {. }
$$

If $\left\{x_{n}\right\}$ is bounded then $\mu \in \mathbb{R}^{+}$and

$$
\lim _{n \rightarrow \infty} f\left(x_{n}\right)=f(\mu) \text {. }
$$

Also (2.33) cannot hold and so (2.34) holds. Then by $(2.19,(2.20),(2.34)$ and (2.35) it follows that $\mu=0$. From this fact and the monotonicity of $\left\{\Delta^{i} x_{n}\right\}$ for $i=0,1$, $\ldots . . \mathrm{m}-1$, it is clear that $(2.29)$ and $(2.30)$ hold.

Next assume that $\left\{x_{n}\right\}$ is unbounded. Then $\mu=\infty$. By (2.35), $\left(2.19^{\circ}\right)$ and (2.20) we see that (2.33) holds and so (2.31) is satisfied. The proof is complete.

COROLLARY 4. Assume that (2.18), (2.19) and $(2.20)$ hold and that $m$ is odd. Then every bounded solution of Eq.(2.28) oscillates.

3. COMPARISON THEOREMS FOR EQUATIONS (1.1) AND (1.2).

In this section we will establish some comparison theorecs for the oscillation of the difference equations $(1,1)$ and $(1.2)$.

THEOREM 1. Assume that $m$ is odd, (1.3), (2.19), (2.19') and (2.20) hold, $f$ or $B$ 1s nondecreasing in some neighborhood of the origin,

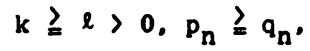

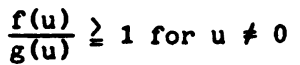

and

$$
\sum_{i=n+1}^{n+l} a_{1}>0 \text { for } n \text { sufficiently large. }
$$

Suppose also that every solution of Eq.(1.2) oscillates. Then every solution of Eq. (1.1) also oscillates.

PROOF. Assume, for the sake of contradiction, that Eq.(1.1) has a nonoscillatory 
solution $\left\{x_{n}\right\}$. We will assume that $\left\{x_{n}\right\}$ is eventually positive. The case where $\left(x_{n}\right\}$ is eventually negative is similar and will be omitted. By lemma 6 , we see that (2.21) and (2.22) hold. Hence, there exist a positive number $\sigma$ and a positive integer $n_{1}$ such that $0<x_{n} \leqq \sigma$ for $n \geqq n_{1}-k$ and $f$ or $\sigma$ is nondecreasing in $[0, \sigma]$. Then by usins (3.1) and (3.2) we obtain

$$
\Delta \Delta^{m} x_{n}+q_{n} g\left(x_{n-\ell}\right) \leqq 0, n \stackrel{?}{=} n_{1} .
$$

By sumining both sides of this inequality from $m$ to infinity and by using (2.22) we find

$$
-\Delta^{m-1} x_{n}+\sum_{i_{1}=n}^{\infty} q_{i_{1}} g\left(x_{i_{1}-\ell}\right) \leqq 0 .
$$

By repeating the same procedure $n$ times and by noting the fact that $m$ is odd we are led to the inequality

$$
\sum_{1_{2}=n}^{\infty} \sum_{i_{2}=1_{2}}^{\infty} \cdots \sum_{1_{m}=i_{m-1}}^{\infty} a_{1_{m}} g\left(x_{1_{m}-l}\right) \leqq x_{n}, n \geqq n_{1} .
$$

Clearly $\left\{x_{n}\right\}$ is a decreasing positive sequence which tends to zero as $n \rightarrow \infty$. Hence by Lemma 5 the equation

$$
\sum_{1_{1}=n}^{\infty} \sum_{1_{2}=i_{1}}^{\infty} \cdots \sum_{i_{m}=i_{m-1}}^{\infty} a_{1_{m}} g\left(y_{1_{m}-l}\right)=y_{n}
$$

has a positive solution $\left\{y_{n}\right\}_{n=n_{1}-\ell}^{\infty}$. This implies that $\left\{y_{n}\right\}_{n=n_{2}-\ell}^{\infty}$ is also a positive solution of Eq.(1.2) which contradicts the hypothesis that every solution of Eq.(1.2) oscillates. The proof is complete.

By Remark 1 and by an argument similar to that in the proof of Theorem 1 we are led to the following result.

THEOREM 1'. Assume that $m$ is odd, (1.3), (2.19) and (2.20) hold and that (3.1)-(3.3) are satisfied. Suppose also that every bounded solution of Eq.(1.2) oscillates. Then every bounded solution of Eq.(1.1) also oscillates.

THEOREM 2. Assume that $\mathrm{m}$ is even, (1.3), (2.19), (2.20) and (3.1)-(3.3) hold. Suppose also that every bounded solution of Eq.(1.2) oscillates. Then every bounded solution of Eq.(1.1) also oscillates.

PROOF. Otherwise, Eq. (1.1) has a bounded nonoscillatory solution $\left\{x_{n}\right\}$. We will assume that $\left\{x_{n}\right\}$ is eventually positive. The case where $\left\{x_{n}\right\}$ is eventually negative is similar and will be omitted.

By Lemma 7, (2.29) and (2.30) hold. Hence, there exist a positive number $\sigma$ and a positive integer $n_{1}$ such that $0<x_{n} \leqq$ for $n \geqq n_{1}-k$ and $f$ or $g$ is nondecreasing in $[0, \sigma]$. Then by using (3.1) and (3.2) we obtain

$$
\Delta^{m} x_{n}-q_{n} g\left(x_{n-l}\right) \geqq 0 \text { for } n \stackrel{?}{=} n_{1} \text {. }
$$

By summing both sides of this inequality $m$ times from $n$ to infinity, by using (2.30) and by noting the fact that $m$ is even we are led to the inequality

$$
\sum_{i_{1}=n}^{\infty} \sum_{i_{2}=i_{1}}^{\infty} \cdots \sum_{i_{m}=i_{m-1}}^{\infty} q_{i_{m}} g\left(x_{i_{m}-l}\right) \leqq x_{n} .
$$


As $\left\{x_{n}\right\}_{n=n_{1}-\ell}^{\omega}$ is a decreasing positive sequence and $\lim _{n \neq \infty} x_{n}=0$, it follows from Leara 5 that the equation

$$
\sum_{i_{1}=n}^{\infty} \sum_{i_{2}=i_{1}}^{\infty} \cdots \sum_{i_{n}=i_{m-1}}^{\infty} q_{i_{m}} \ddot{i}\left(y_{i_{L,}-\ell}\right)=y_{n}
$$

has a positive solution $\left\{y_{n}\right\}_{n=n_{1}-l}^{\infty}$ such that $\operatorname{lin}_{n \neq \infty} y_{n}=0$. Clearly this sequence is also a bounded positive solution of Eq.(1.2) which contradicts the hypothesis that every bounded solution of Eq.(1.2) oscillates. The proof of the theorem is complete.

4. LINEARIZED OSCILLATIONS.

In this section we will establish linearized oscillation theorems for Eq.(1.1) with m odd or even.

THEOREM 3. Assume that $m$ is odd, (2.18), (2.19) and (2.19') hold.

$$
\liminf _{n \rightarrow \infty} p_{n}=p \in(0, \infty)
$$

and that

$$
\liminf _{u \rightarrow 0} \frac{f(u)}{u} \geqq 1
$$

Suppose that every solution of Eq.(1.4) oscillates. Then every solution of Eq.(1.1) also oscillates.

PROOF. Assume, for the sake of contradiction, that Eq.(1.1) has a nonosc1llatory solution $\left\{x_{n}\right\}$. We will assune that $\left\{x_{n}\right\}$ is eventually positive. The case where $\left\{x_{n}\right\}$ is eventually negative is similar and will be omitted. By Lemma 6, (2.21) and (2.22) hold. Now for $n$ sufficiently large we rewrite Eq.(1.1) in the form

$$
\Delta^{m} x_{n}+P_{n} x_{n-k}=0
$$

where

Note that

$$
p_{n}=p_{n} \frac{f\left(x_{n-k}\right)}{x_{n-k}}
$$

$$
\liminf _{n \rightarrow \infty} P_{n} \geq p
$$

Then for any positive number e in the interval $0<\&<(1 / 2) p$. Eq.(4.3) yields, for n sufficiently large,

$$
\Delta^{m} x_{n}+(p-e) x_{n-k} \leqq 0
$$

By summing both sides of this inequality $m$ times from $n$ to infinity, by using (2.22) and by noting the fact that $m$ is odd we find

$$
(p-\varepsilon) \sum_{1_{2}=n}^{\infty} \sum_{i_{2}=1_{1}}^{\infty} \ldots \sum_{i_{m}=i_{m-1}}^{\infty} x_{i_{m}-k} \leqq x_{n}, n \geqq N
$$

where $N$ is sufficiently large and $\left\{x_{n}\right\}$ is a decreasing positive sequence which tends to zero as $n \rightarrow \infty$. Then by Lemma 5 we see that the equation 


$$
(p-\varepsilon) \sum_{i_{1}=n}^{\infty} \sum_{i_{2}=1}^{\infty} \cdots \sum_{i_{m}=i_{m-1}}^{\infty} v_{i_{m}-k}=v_{n}
$$

has a positive solution $\left\{v_{n}\right\}$ for $n ? N-k$. It is easy to see that $\left\{v_{n}\right\}$ is also a positive solution of the difference equation

$$
\Delta^{m} v_{n}+(p-\varepsilon) v_{n-k}=0 \text {. }
$$

Hence, by Lemma 3 and because of the fact that the $\varepsilon$ is arbitrarily small, it follows that Eq. (1.4) has a positive solution. This contradicts the hypothesis and completes the proof of the theorem.

THEOREY 4. Assume that $m$ is even, (2.18), (2.19), (4.1) and (4.2) hold and that every bounded solution of Eq.(1.4) oscillates. Then every bounded solution of Eq. (1.1) also oscillates.

PROOF. Otherwise there exists a bounded nonoscillatory solution $\left[x_{n}\right\}$ of Eq.(1.1). We will assume that $\left\{x_{n}\right\}$ is eventually positive. The case where $\left\{x_{n}\right\}$ is eventually negative is similar and will be omitted. By Lemma 7, (2.29) and (2.30) hold. Now for n sufficiently large we rewrite Eq.(1.1) in the form

$$
\Delta x_{n}-P_{n} x_{n-k}=0
$$

where

Note that

$$
P_{n}=\frac{f\left(x_{n-k}\right)}{x_{n-k}}
$$

$$
\liminf _{n \rightarrow \infty} P_{n} \stackrel{p}{p}
$$

Then for any positive $\varepsilon$ in the interaval $0<\varepsilon<(1 / 2) p$, Eq.(4.5) yields

$$
\Delta x_{n}^{m}-(p-\varepsilon) x_{n-k} \geq 0 \text {. }
$$

By an argument similar to that in the proof of Theorem 3 and by noting that $m$ is even we see that the difference equation

$$
\Delta^{m} v_{n}-(p-\varepsilon) v_{n-k}=0
$$

has a bounded positive solution. Hence, by Lemma 4 and because of the fact that $e$ is arbitrarily small, it follows that Eq.(1.4) has a bounded positive solution. This contradicts the hypothesis and completes the proof of the theorem.

\section{EXISTENCE OF POSITIVE SOLUTIONS}

The next theorem is a partial converse of Theorem 3 and shows that, under appropriate hypotheses, Eq.(1.1) has a positive solution provided that an associated linear equation also has a positive solution.

THEOREM 5. Assume that in is odd, (2.18) holds and suppose that there exist positive constants $p$ and $\delta$ such that

$$
0 \leqq p_{n} \leqq p
$$

and that

either $0 \leqq f(u) \leqq u$ for $0 \leqq u \leqq \delta$

or $\quad 0 \geqq f(u) \geqq u$ for $-\delta \leqq u \leqq 0$. 
Suppose also that

$f(u)$ is nondecreasing in a neighborhood of the origin and that the characteristic equation of Eq.(1.4)

$$
(\lambda-1)^{\mathrm{m}}+p \lambda^{-k}=0
$$

has a positive root. Then Eq.(1.1) has a nonoscillatory solution.

PROOF. Assume that $0 \leqq f(u) \leqq u$ for $0 \leqq u \leqq \delta$ holds. The case where $0 \geqq f(u) \geqq$ $u$ for $-\delta \leqq u \leqq 0$ holds is similar and will be omitted. Let $\lambda_{0}$ be a positive root of Eq.(5.4). Then it is easy to see that $\lambda_{0}<1$. Set $z_{n}=\lambda_{0}{ }^{n}$. Then there exists a N $\stackrel{x}{k}$ such that

$$
0<z_{n} \leqq \delta \text { for } n \geqq n-k
$$

and $f$ is nondecreasing in $\left[0, z_{N-k}\right]$. Clearly,

$$
\begin{aligned}
& z_{n}>0, \Delta z_{n}\left\langle 0, \Delta^{2} z_{n}>0, \ldots . \Delta^{m-1} z_{n}>0, \Delta^{m} z_{n}<0,\right. \\
& \lim _{n \rightarrow \infty} \Delta^{i} z_{n}=0 \text { for } i=0,1, \ldots m .
\end{aligned}
$$

and $\left(z_{n}\right)$ satisfies the difference equation

$$
\Delta \mathbf{z}_{\mathrm{n}}+\mathrm{p} \mathbf{z}_{\mathrm{n}-\mathrm{k}}=\mathbf{0}
$$

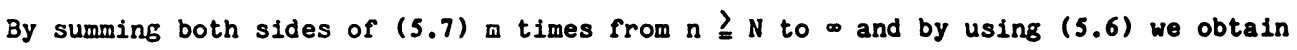

$$
\sum_{1_{1}=n}^{\infty} \sum_{i_{2}=i_{2}}^{\infty} \ldots \sum_{i_{m}=i_{m-1}}^{\infty} p z_{i_{m}-k}=z_{n}, n \geqq N \text {. }
$$

In view of (5.1) and (5.2), this equation implies that

$$
\sum_{i_{1}=n}^{\infty} \sum_{i_{2}=i_{2}}^{\infty} \ldots \sum_{i_{m}=i_{m-1}}^{\infty} p_{i_{m}} f\left(z_{i_{m}-k}\right) \leqq z_{n}, n \geqq N .
$$

Then from Lemma 5 it follows that the corresponding equation

$$
\sum_{i_{1}=n}^{\infty} \sum_{i_{2}=i_{1}}^{\infty} \ldots \sum_{i_{m}=i_{m-1}}^{\infty} p_{i_{m}} f\left(x_{i_{m}-k}\right)=x_{n}, n \geqq N
$$

has a positive solution. This implies that Eq.(1.1) has a positive solution. The proof of the theorem is complete.

By combining Theorems 2 and 4 we obtain the following necessary and sufficient condition for the oscillation of every solution of Eq.(1.1) when $m$ is even.

COROLLARY 4. Assume that $m$ is even and that there exist positive constant $c_{0}, p$ and $\delta$ such that (22), (2.19), (2.19\%), (5.2) and (5.3) hold and that

$$
0 \leqq p_{n} \leqq p=\lim _{n \rightarrow \infty} p_{n} \text {, and } \lim _{u \rightarrow 0} \frac{f(u)}{u}=1 \text {. }
$$

Then every solution of Eq.(1.1) oscillates if and only if the equation

$$
(\lambda-1)^{m}-p \lambda^{-k}=0
$$

has no positive roots.

The next theorem is a partial converse of Theorem 4 and shows that, under appropriate hypotheses. Eq.(1.1) has a bounded positive solution provided that an associated linear equation also has a bounded positive solution.

THEOREM 6. Assume that $m$ is even, (2.18) and (5.1)-(5.3) hold. Suppose also that the characteristic equation (5.9) of Eq.(1.4) has a real root in the interval $(0,1)$. Then Eq.(1.1) has a bounded nonoscillatory solution. 
PROOF. Assume that $0 \leqq f(u) \leqq u$ for $0 \leqq u \leqq \sigma$. The case where $0 \leqq f(u) \geqq u$ for $-\delta \leqq u$ $\leqq 0$ is similar and will be omitted. Let $\lambda_{0}$ be a root of Eq.(5.9) in the interval (0, 1). Set $z_{n}=\lambda_{0}{ }^{n}$. Then there exists a $N \stackrel{0}{=}$ such that

$$
0<z_{n} \leqq \delta \text { for } n \geqq N-k
$$

and $f$ is nondecreasing in $\left[0, z_{N-k}\right]$. Clearly,

$$
\begin{aligned}
& z_{n}>0, \Delta z_{n}\left\langle 0, \Delta^{2} z_{n}\right\rangle 0, \ldots . \Delta^{m-1} z_{n}\left\langle 0, \Delta^{m} z_{n}\right\rangle 0, \\
& \lim _{n \rightarrow \infty} \Delta^{i} z_{n}=0 \text { for } i=0,1,2, \ldots . m
\end{aligned}
$$

and $\left\{z_{n}\right\}$ satisfies the difference equation

$$
\Delta z_{n}-p z_{n-k}=0 \text {. }
$$

Then by noting that $m$ is even and by an argument similar to that in the proof of Theorem 5 we find that Eq.(1.1) has a bounded positive solution. The proof of the theorem is complete.

Finally by combining Theorem 4 and 6 we obtain the following necessary and sufficient condition for the oscillation of every bounded solution of Eq.(1.1) when $n$ is even.

CORULLARY 5. Assume that $m$ is even, and that there exist positive constants $p$ and $\delta$ such that $(2.18),(2.19),(5.2),(5.3)$ and $(5.8)$ hold. Then every bounded solution of Eq. (1.1) oscillates if and only if $\mathrm{Eq} .(5.9)$ has no real roots in the interval $(0,1]$.

\section{REFERENCES}

1. ERBE, L. H. and ZHANG, B. G. Oscillation of discrete analogues of delay equations, Proceeding of the International Conference on Theory and Applications of Differential Equations, March 21-25, 1988, Ohio University, to be published by Marcel Dekker, Inc.

2. GYÖRI, I. and LADAS, G. Linearized oscillations for equations with piecewise constant arguments, Differentiala and Integ $\underline{\text { ral }}$ Eq Equatíions 2(1989), 123-131.

3. GYŌRI, I. , LADAS, G. and PAKULA, L. Conditions for the oscillation of difference equations with applications to equations with piecewise constant arguments (to appear).

4. HOOKER, JOHN W. and PATULA, William T. A second-order nonlinear difference

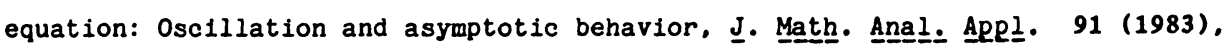
9-29.

5. LADAS, G. Oscillations of equations with piecewise constant mixed arguments, International Conference on Theory and Applications of Differential Equations. March 21-25, 1988, Ohio University, to be published by Marcel Dekker, Inc.

6. LADAS, G. Oscillations of difference equations with positive and negative coefficients, Geoffrey J. Butler Memorial Conference on Differential Equations and Population Biology, June 20-25, 1988, University of Alberta, Edmonton, Canada.

7. LADAS, G., PHILOS, Ch. G. and SFICAS, Y. G. Sharp conditions for the oscillation

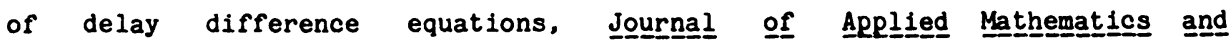


Singula

8. MINGARELLI, A. B. Volterra-Stieltjes integral equations and generalized differential equations, Ph. D. Dissertation, University of Toronto, 1979.

9. PATULA, W. T. Growth, oscillation and comparison theorems for second order linear difference equations, SIAll J. Math. Anal. 10(6) 1979, 1272-1279.

10. PHILOS, Ch. G. and SFICAS, Y. G. Positive solutions of difference equations (to appear). 


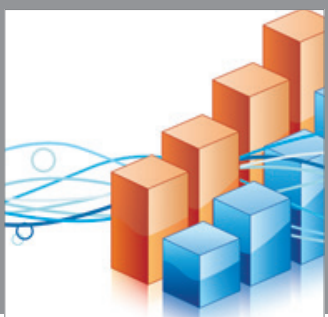

Advances in

Operations Research

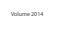

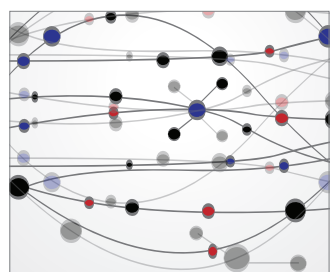

\section{The Scientific} World Journal
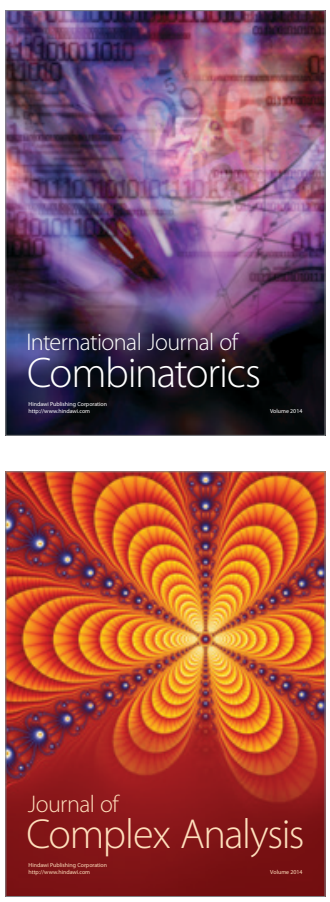

International Journal of

Mathematics and

Mathematical

Sciences
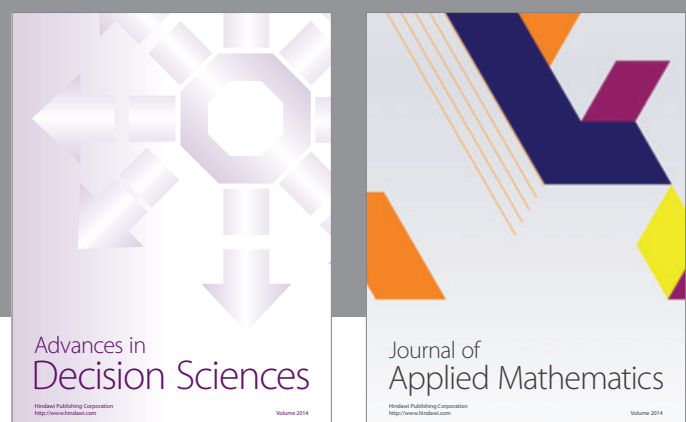

Journal of

Applied Mathematics
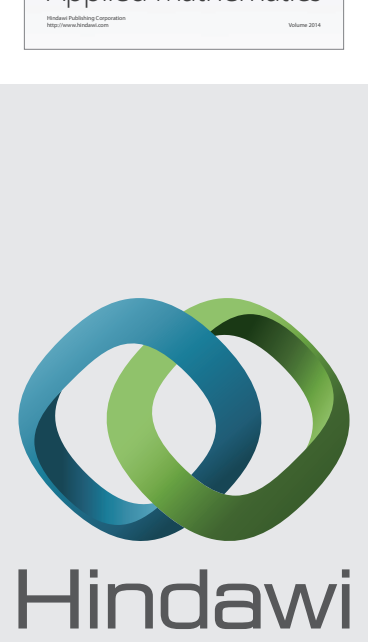

Submit your manuscripts at http://www.hindawi.com
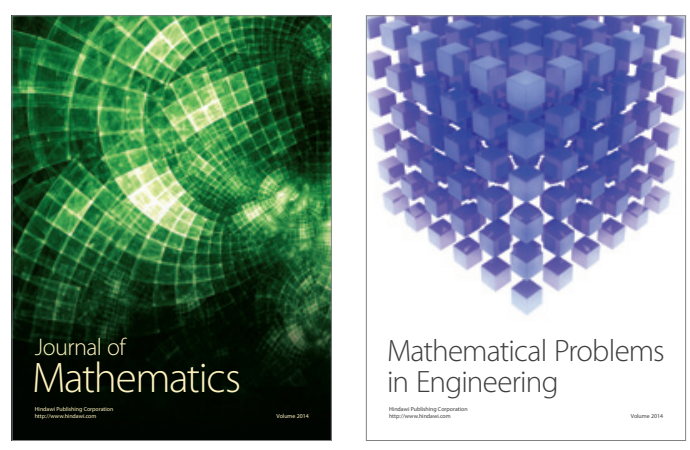

Mathematical Problems in Engineering
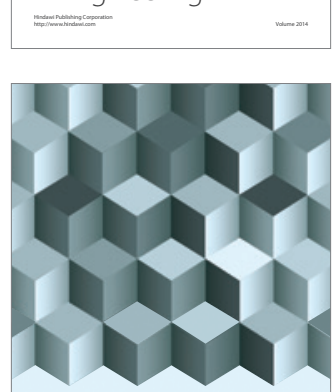

Journal of

Function Spaces
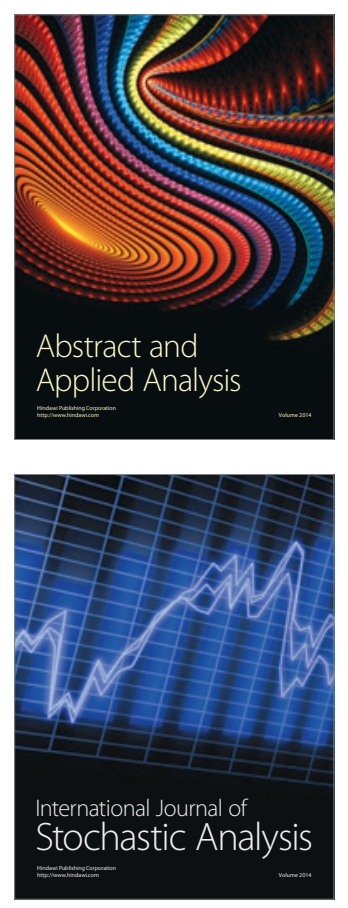

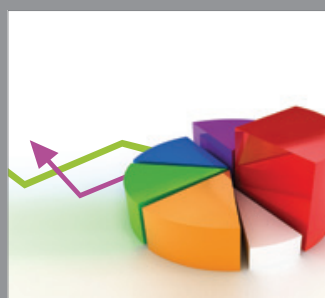

ournal of

Probability and Statistics

Promensencen
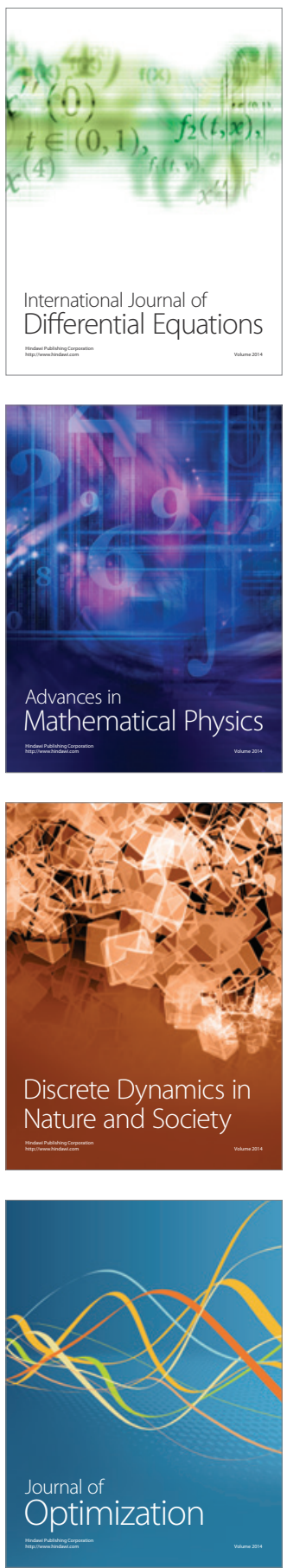